\title{
Consumo alimentar de crianças e adolescentes com disfagia decorrente de estenose de esôfago: avaliação com base na pirâmide alimentar brasileira
}

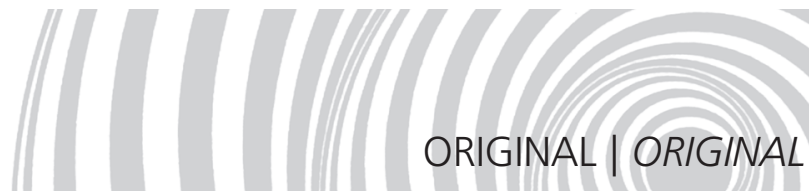

Food consumption by children and adolescent with

dysphagia due to esophageal stricture: assessment

based on the Brazilian food guide pyramid

Renata MARCIANO'

Patrícia da Graça Leite SPERIDIÃO2,3

Elisabete KAWAKAMI ${ }^{3}$

RE S U M O

\section{Objetivo}

Avaliar o consumo alimentar de pacientes com disfagia decorrente de estenose de esôfago, comparando a dieta de consistência líquida com a dieta de consistência pastosa e sólida, com base na Pirâmide Alimentar Brasileira.

\section{Métodos}

Estudo de corte transversal, no qual foram incluídos consecutivamente 31 pacientes com estenose esofágica, sendo 18 (58,0\%) cáustica, 7 (22,6\%) pós-cirúrgica, 3 (9,7\%) péptica e 3 (9,7\%) sem causa definida. Empregou-se o recordatório de 24 horas; os alimentos foram transformados em porções em função dos oito grupos de alimentos, conforme recomendado por Philippi. Utilizou-se o teste Kruskal-Wallis e Exato de Fisher, fixando em $5 \%$ o nível de rejeição da hipótese de nulidade.

\section{Resultados}

A idade variou entre 15 e 176 meses (mediana, 56 meses), sendo 28 crianças e três adolescentes, e 18 do sexo masculino. Vinte e nove pacientes (93,5\%) apresentavam disfagia, sendo grave em 34,4\% (10/29), moderada em $41,3 \%$ (12/29), e leve em 24,1\% (7/29). O consumo mediano de porções de cereais, leguminosas, e óleos

$\overline{1}$ Universidade Federal de São Paulo, Programa de Pós-Graduação em Pediatria e Ciências Aplicadas em Pediatria. R. Pedro de Toledo, 441, Vl. Clementino, 04039-031, São Paulo, SP, Brasil. Correspondência para/Correspondence to: R. MARCIANO. E-mail: <renata.marciano@bol.com.br>.

2 Universidade Federal de São Paulo, Departamento de Pediatria, Disciplina de Gastroenterologia Pediátrica. São Paulo, SP, Brasil.

${ }^{3}$ Universidade Federal de São Paulo, Curso de Nutrição, Baixada Santista. Santos, SP, Brasil. 
234 | R. MARCIANO et al.

e gorduras foi menor no grupo com dieta líquida $(p<0,005)$, o qual também apresentou maior proporção de pacientes cujo consumo foi abaixo do proposto pela pirâmide alimentar quando comparado ao grupo com dieta pastosa e sólida, com diferença estatisticamente significante $(p<0,05)$.

\section{Conclusão}

O suporte nutricional é de extrema importância no tratamento de pacientes com estenose esofágica, principalmente na disfagia grave, cuja dieta deve ser adaptada à consistência líquida, devido ao risco nutricional que se atribui à limitada ingestão alimentar, e para que o tratamento dietético seja precocemente instituído.

Termos de indexação: Consumo alimentar. Estenose esofágica. Pirâmide alimentar. Transtornos de deglutição.

\section{A B S T R A C T}

\section{Objective}

This study assessed food intake by patients with dysphagia due to esophageal stricture and compared liquid, soft and solid diets based on the Brazilian Food guide pyramid.

\section{Methods}

This cross-sectional study consecutively included 31 patients with esophageal stricture, of which 18 (58.0\%) were caustic, 7 (22.6\%) were postoperative, 3 (9.7\%) were peptic and 3 (9.7\%) were of unknown etiology. The 24-hour dietary recall was used and the foods were converted into servings according to the eight food groups, as recommended by Philippi. The Kruskal-Wallis and Fisher's Exact Test were used and the significance level was set at 5\%.

\section{Results}

The ages of the patients varied from 15 to 176 months (median: 56 months). There were 28 children and 3 adolescents, of which 28 were males. Twenty-nine patients (93.5\%) presented dysphagia, of which $34.4 \%$ (10/29) were severe, $41.3 \%$ (12/29) were moderate and $24.1 \%$ (7/29) were mild. The median intake of grain, legume and fat servings was smaller in the liquid diet group $(p<0.005)$. This group also had a significantly greater proportion of patients whose intakes were below those recommended by the food pyramid $(p<0.05)$.

\section{Conclusion}

Nutritional support is extremely important in the treatment of patients with esophageal stricture, especially those with severe dysphagia. These patients need a liquid diet because of the nutritional risk associated with inadequate food intake, which also allows early introduction of the dietary treatment.

Indexing terms: Food consumption. Esophageal stenosis. Food pyramid. Deglutition disorders.

\section{N T R O D U Ç Ã O}

Nas estenoses esofágicas, em que a alteração anatômica do esôfago resulta em considerável diminuição do lúmen, a disfagia é considerada a principal manifestação clínica, ocorrendo inicialmente com os alimentos sólidos, evoluindo para líquidos conforme a evolução da fibrose ${ }^{1}$.

A estenose benigna de esôfago desenvolve-se após uma lesão na parede do órgão, resultando no desenvolvimento da fibrose. Pode ser causada pela ingestão de álcalis ou ácidos, ou ainda pela complicação de esofagite péptica ou eosinofílica, após intervenção cirúrgica de esôfago, pós-escleroterapia de varizes esofágicas, entre outros. A estenose secundária à correção cirúrgica de atresia de esôfago ocorre entre 30\% a 50\% das crianças, e pode se desenvolver entre um a sete meses após a cirurgia22-4. A estenose péptica é a complicação mais frequente da esofagite de refluxo, e seu diagnóstico geralmente ocorre tardiamente, em média após dois anos do início do sintomas ${ }^{5-7}$. A ingestão de substância cáustica é o principal fator exógeno desencadeante da estenose esofágica entre as crianças ${ }^{8,9}$, e seu desenvolvimento está relacionado à gravidade da injúria esofageana, uma vez que cerca de $20 \%$ das crianças que ingerem produto cáustico apresentam lesões esofagianas, e 20\% a 65\% delas desenvolvem estenose esofágica ${ }^{10,11}$. A maioria dos 
acidentes ocorre no próprio domicílio devido à facilidade de acesso aos produtos químicos e pela falta de informação à população a respeito das graves consequências advindas da ingestão desses produtos $^{12}$.

A dilatação com sondas de Savary-Gilliard é amplamente empregada no tratamento da estenose e visa a atenuar o sintoma disfágico, o que facilita a ingestão alimentar ${ }^{13-15}$. No entanto, a recorrência de disfagia é frequente ${ }^{14}$, fazendo com que constantes modificações na consistência da dieta sejam realizadas a fim de possibilitar o processo de deglutição. Independentemente da etiologia, os pacientes com estenose esofágica estão constantemente submetidos a um quadro de risco nutricional, não apenas pelo estreitamento da luz esofágica, mas também pelo início tardio do tratamento com dilatação, podendo apresentar disfagia grave e sinais de desnutrição, fato descrito na literatura ao longo de 70 anos ${ }^{16,17}$.

A dieta de consistência modificada pode resultar em ingestão alimentar insuficiente e inadequada, principalmente pela falta de orientação nutricional específica, acarretando maior morbidade pelo comprometimento do estado nutricional de crianças e adolescentes com estenose esofágica. Embora o alívio da disfagia e o aumento da ingestão alimentar sejam os principais objetivos da terapêutica da estenose, não há estudos que abordem a ingestão alimentar via oral nem o aspecto qualitativo, ou seja, que ressaltem a composição da dieta de consistência adaptada.

A pirâmide alimentar, amplamente reconhecida como ferramenta de educação nutricional, tem sido útil no monitoramento nutricional, possibilitando verificar as diferenças entre o consumo alimentar atual e o recomendado, e tem respaldado muitos estudos cuja proposta é analisar qualitativamente a dieta ${ }^{18-21}$.

Portanto, este estudo teve como objetivo avaliar qualitativamente o consumo alimentar de pacientes com disfagia decorrente de estenose de esôfago, comparando a dieta de consistência líquida com a dieta de consistência pastosa e sólida, com base na pirâmide alimentar brasileira.

\section{M É T O D O S}

Estudo observacional, de corte transversal, no qual foram incluídos consecutivamente 31 pacientes ambulatoriais com diagnóstico de estenose esofágica, independentemente da etiologia, sendo 28 crianças (menores de dez anos) e três adolescentes.

Para coleta de dados, empregou-se questionário clínico-demográfico na primeira consulta com a nutricionista pesquisadora, durante o período de junho de 2004 a março de 2007. O período entre o diagnóstico e a primeira consulta nutricional variou entre 2 e 144 meses, com mediana de 21 meses (percentil 25, 8,5 meses, e percentil 75, 50,2 meses).

Pacientes com estenose esofágica associada a complicações neurológicas ou síndromes genéticas foram excluídos por apresentarem outras complicações - além da disfagia - que influenciam a ingestão alimentar. Foram excluídos também aqueles que já haviam sido previamente orientados por nutricionista, pois impossibilitaria identificar as possíveis inadequações alimentares, e também aqueles que se alimentavam via sonda nasogástrica ou gastrostomia.

Empregou-se o recordatório de 24 ho$\operatorname{ras}^{22,23}$, a partir do qual se verificaram o horário das refeições, o tipo de preparação (ressaltando a consistência dos alimentos), os alimentos que foram ingeridos e as respectivas quantidades (em gramas, mililitros, ou medida caseira).

As medidas relatadas no recordatório alimentar de 24 horas foram transformadas em porções de acordo com os oito grupos de alimentos distribuídos na pirâmide alimentar: grupo de cereais, pães, tubérculos e raízes; grupo de vegetais; grupo de frutas; grupo de carnes e ovos; grupo de leguminosas; grupo de leite e derivados; grupo de óleos e gorduras; grupo de açúcares.

Considerou-se "porção" a quantidade de alimento em sua forma usual de consumo, expressa em medidas caseiras (xícara, colher de sopa), unidades ou na forma de consumo. 
O consumo de porções de cada grupo alimentar foi comparado ao número de porções proposto por três pirâmides alimentares ${ }^{19,24,25}$, segundo a faixa etária, adaptadas para a população brasileira (Quadro 1).

A consistência da dieta foi classificada em: líquida, quando a dieta era composta de alimentos que se liquefazem na boca, ou liquidificados em consistência rala ou coados; pastosa quando os alimentos eram consumidos amassados, umidificados ou triturados; e sólida, quando os alimentos eram ingeridos sem adaptação em sua consistência.

Uma vez que foi identificada a consistência de dieta tolerada pelo paciente, a disfagia foi classificada em: ausência de disfagia; disfagia leve, quando se manifestava ocasionalmente a alguns alimentos sólidos, não sendo necessárias adaptações na consistência para deglutição; disfagia moderada, quando se manifestava aos alimentos sólidos, no qual os pacientes toleravam consistência pastosa e líquida; disfagia grave, em que os pacientes toleravam exclusivamente líquidos.

Foi utilizado o teste Kruskal-Wallis, e quando revelou diferença estatisticamente significante, a análise foi complementada com o teste de Dunn.

O teste exato de Fisher foi utilizado para verificar a associação da consistência da dieta ao número (\%) de pacientes cuja ingestão de porções

Quadro 1. Número de porções recomendado para cada grupo de alimento pela pirâmide alimentar, segundo a faixa etária. São Paulo (SP), 2007.

\begin{tabular}{|c|c|c|c|}
\hline \multirow[t]{2}{*}{ Grupos de alimentos } & \multicolumn{3}{|c|}{$\begin{array}{c}\text { Número de porções recomendado } \\
\text { por faixa etária }\end{array}$} \\
\hline & $<2$ anos $^{*}$ & 2 a 3 anos $^{* *}$ & $>3 \operatorname{anos}^{\star \star \star}$ \\
\hline Cereais & 3 a 5 & 5 & 5 a 9 \\
\hline Vegetais & 3 & 3 & 4 a 5 \\
\hline Frutas & 3 a 4 & 3 & 3 a 5 \\
\hline Leite e derivados & 3 & 3 & 3 \\
\hline Carnes e ovos & 2 & 2 & 1 a 2 \\
\hline Leguminosas & 1 & 1 & 1 \\
\hline Açúcares & 1 & 1 & 1 a 2 \\
\hline Gorduras & 2 & 1 & 1 a 2 \\
\hline
\end{tabular}

${ }^{*}$ Monte et al. ${ }^{24 ;}$; ${ }^{* *}$ Philippi et al. ${ }^{19}$; ${ }^{* \star *}$ Philippi et al. ${ }^{25}$. estava abaixo do proposto pela pirâmide alimentar.

Para os cálculos estatísticos utilizou-se o programa de computador Sigma Stat. 2.026. Fixou-se em $5 \%(\alpha \leq 0,05)$ o nível de rejeição da hipótese de nulidade.

O estudo foi aprovado pelo Comitê de Ética em Pesquisa da Universidade Federal de São Paulo - Escola Paulista de Medicina (CEP n 0876/05), e todos os participantes assinaram o termo de consentimento livre e esclarecido antes de sua inclusão na amostra.

\section{RESULTADOS}

Foram avaliadas 28 crianças (menores de 10 anos) e 3 adolescentes, sendo 18 do sexo masculino (58\%). A idade variou entre 15 e 176 meses (mediana, 56 meses; percentis 25 e 75, 28,2 e 68,5 meses, respectivamente). A Tabela 1 apresenta as características demográficas dos pacientes segundo a etiologia da estenose esofágica.

Ausência de disfagia foi observada em 6,5\% (2/31) dos pacientes, e 93,5\% (29/31) apresentavam disfagia em graus variados, destes, $24,1 \%(7 / 29)$ apresentavam disfagia leve, 41,3\% (12/29) disfagia moderada, e 34,4\% (10/29) apresentavam disfagia grave. Os pacientes foram classificados em 3 grupos, de acordo com a consistência da dieta, sendo dieta líquida $(n=10)$, dieta pastosa $(n=12)$ e dieta sólida ( $n=9)$.

Dentre os pacientes que estavam no programa de dilatação $(n=27)$, foi observado que aqueles com disfagia leve ou ausente haviam realizado maior número de sessões de dilatação do que os pacientes com disfagia grave. $\mathrm{O}$ diâmetro do dilatador (em milímetros) empregado na última sessão de dilatação também foi maior entre os pacientes com disfagia leve ou ausente, com significância estatística (Tabela 2).

Independentemente da consistência da dieta, observou-se que o consumo de porções foi inferior ao proposto pela pirâmide alimentar para os seguintes grupos alimentares: cereais, pães, tubérculos e raízes em 64,5\% (20/31) dos pacien- 
tes; grupo de vegetais em 93,5\% (29/31); frutas em 70,9\% (22/31); grupo de leite e derivados em $45,1 \%$ (14/31); grupo de carnes e ovos em $58,0 \%$ (18/31); grupo de leguminosas em 41,9\% (13/31); grupo de açúcares em 6,4\% (2/31); e grupo de óleos e gorduras em 74,1\% (23/31) dos pacientes.

A ingestão mediana de porções do grupo de cereais e do grupo óleos e gorduras foi menor entre os pacientes com dieta líquida, com significância estatística quando comparada ao grupo com dieta pastosa e sólida (Tabela 3). Em relação ao grupo de frutas, a ingestão foi menor no grupo com dieta líquida quando comparado ao grupo com dieta sólida, com diferença estatisticamente significante. Quanto ao grupo de leguminosas, a ingestão foi menor entre os pacientes com dieta líquida, em comparação ao grupo com dieta pastosa. Quanto ao grupo de açúcares, notou-se que a ingestão foi maior na dieta pastosa, quando comparado ao grupo com dieta sólida, com significância estatística (Tabela 3).

No grupo com dieta líquida foi maior a proporção de pacientes cujo consumo de porções do grupo de cereais, leguminosas e óleos e gorduras estava abaixo do proposto pela pirâmide alimentar, com significância estatística (Tabela 4).

Tabela 1. Características demográficas dos 31 pacientes com estenose de esôfago. São Paulo (SP), 2007.

\begin{tabular}{|c|c|c|c|c|c|}
\hline \multirow{2}{*}{ Etiologia da estenose } & \multirow{2}{*}{$\mathrm{n}$} & \multirow{2}{*}{$\%$} & \multicolumn{2}{|c|}{ Idade (meses) } & \multirow{2}{*}{$\frac{\text { Sexo }}{\text { Masculino - Feminino }}$} \\
\hline & & & Mediana & $(p 25-p 75)$ & \\
\hline Cáustica & 18 & 58,0 & 62,0 & $(38,0-79,0)$ & $13: 5$ \\
\hline Pós-cirúrgica & 7 & 22,6 & 29,0 & $(26,2-34,7)$ & $3: 4$ \\
\hline Péptica & 3 & 9,7 & 60,0 & $(57,0-60,7)$ & $1: 2$ \\
\hline Outras & 3 & 9,7 & 27,0 & $(18,0-79,5)$ & $1: 2$ \\
\hline Total & 31 & 100,0 & 56,0 & $(28,2-68,5)$ & $18: 13$ \\
\hline
\end{tabular}

Tabela 2. Número de sessões de dilatação esofágica, e diâmetro do dilatador alcançado, segundo os graus de disfagia (n=27). São Paulo (SP), 2007.

\begin{tabular}{lcccc}
\hline & & Graus de disfagia & & \\
\cline { 2 - 4 } & Ausente e leve $n=9$ & Moderada $n=10$ & Grave $n=8$ & $2(1-6)$ \\
\hline Número de sessões de dilatação & $17(4,7-26,2)$ & $3,5(1-7)$ & $0,018^{1}$ \\
Diâmetro do dilatador (mm) & $12,8(11,7-12,85)$ & $10,5(9-11)$ & $7(5,5-10,1)$ & $<0,001^{2}$ \\
\hline
\end{tabular}

Mediana e percentis 25 e 75 entre parênteses. Teste Kruskal-Wallis; Teste de comparações múltiplas de Dunn, $p<0,05$ em; ${ }^{1}$ Leve e ausente vs grave;

2 Leve e ausente vs grave; leve e ausente vs moderada.

Tabela 3. Ingestão de porções de cada grupo de alimentos da pirâmide alimentar, segundo a consistência da dieta. São Paulo (SP), 2007.

\begin{tabular}{lcccc}
\hline & \multicolumn{3}{c}{ Consistência da dieta } & \multicolumn{1}{c}{ Sólida $(n=9)$} \\
\cline { 2 - 3 } & Líquida $(n=10)$ & Pastosa $(n=12)$ & $5,0(3,6-6,8)$ & $<0,001^{1}$ \\
\hline Cereais & $0,7(0-1,1)$ & $4,9(3,5-7,5)$ & $1,0(0,0-2,5)$ & 0,248 \\
Vegetais & $0,0(0,0-1,0)$ & $0,2(0,0-0,5)$ & $2,0(1,8-6,2)$ & $0,014^{2}$ \\
Frutas & $0,0(0,0-1,0)$ & $1,1(0,0-4,5)$ & $2,4(1,4-3,2)$ & 0,143 \\
Leite e derivados & $3,3(2,2-4,2)$ & $3,4(2,6-4,6)$ & $1,6(0,7-3,0)$ & 0,089 \\
Carnes e ovos & $0,6(0,0-1,0)$ & $0,5(0,0-2,1)$ & $1,4(0,7-2,5)$ & $0,005^{3}$ \\
Leguminosas & $0,0(0,0-0,2)$ & $1,8(1,0-3,0)$ & $1,5(1,0-2,1)$ & $0,018^{4}$ \\
Açúcares & $1,5(1,0-3,0)$ & $2,9(2,1-5,4)$ & $1,0(0,5-4,1)$ & $0,001^{5}$ \\
Óleos e gorduras & $0,0(0,0-0,0)$ & $0,0(0,0-0,6)$ & & \\
\hline
\end{tabular}

Teste de comparações múltiplas de Dunn, p<0,05 em; ${ }^{1}$ líquida vs pastosa, líquida vs sólida; ${ }^{2}$ líquida vs sólida; ${ }^{3}$ líquida vs pastosa; ${ }^{4}$ pastosa vs sólida; 5 líquida vs sólida, pastosa vs sólida.

Mediana e percentils 25 e 75 entre parênteses. Teste Kruskal-Wallis. 
Tabela 4. Número de pacientes cujo consumo de porções estava abaixo do proposto pela pirâmide alimentar segundo a consistência da dieta. São Paulo (SP), 2007.

\begin{tabular}{|c|c|c|c|c|c|c|}
\hline & \multicolumn{6}{|c|}{ Consistência da dieta } \\
\hline & \multicolumn{2}{|c|}{ Líquida ( $\mathrm{n}=10)$} & \multicolumn{2}{|c|}{ Pastosa $(n=12)$} & \multicolumn{2}{|c|}{ Sólida $(n=9)$} \\
\hline & $\mathrm{n}$ & $\%$ & $\mathrm{n}$ & $\%$ & $n$ & $\%$ \\
\hline Cereais $^{1}$ & 10 & 100,0 & 6 & 50,0 & 4 & 44,4 \\
\hline Vegetais & 9 & 90,0 & 12 & 100,0 & 8 & 88,8 \\
\hline Frutas & 10 & 100,0 & 7 & 58,3 & 5 & 55,5 \\
\hline Leite e derivados & 4 & 40,0 & 4 & 33,3 & 6 & 66,6 \\
\hline Carnes e ovos & 8 & 80,0 & 7 & 58,3 & 3 & 33,3 \\
\hline Leguminosas $^{2}$ & 8 & 80,0 & 3 & 25,0 & 2 & 22,2 \\
\hline Açúcares & 1 & 10,0 & 0 & 0,0 & 1 & 11,1 \\
\hline Óleos e gorduras 3 & 10 & 100,0 & 10 & 83,3 & 3 & 33,3 \\
\hline
\end{tabular}

1 líquida vs pastosa $(p=0,015)$, líquida vs sólida $(p=0,011)$, pastosa vs sólida $(p=1,000)$; 2 líquida vs pastosa $(p=0,030)$, líquida vs sólida ( $p=0,023)$, pastosa vs sólida $(p=1,000)$; 3íquida vs pastosa $(p=0,481)$, líquida vs sólida $(p=0,003)$, pastosa vs sólida $(p=0,032)$.

Teste Exato de Fisher (aos pares).

\section{I S C U S S Ã O}

A nutrição adequada é um dos mais importantes aspectos na abordagem do paciente com estenose de esôfago, que diante de inúmeras situações de risco nutricional evolui com depleção de grande parte das reservas corporais, e total limitação do consumo alimentar devido o estreitamento esofágico, porém, estudos sobre avaliação do estado nutricional e consumo alimentar são escassos na literatura científica.

Os resultados permitiram constatar que todos os pacientes com dieta líquida apresentaram consumo inferior ao proposto pela pirâmide alimentar em relação às porções do grupo de cereais, pães, tubérculos e raízes, do grupo de frutas e de óleos de gorduras. Porém, também foi observado que o consumo do grupo de vegetais e de carnes e ovos não foi baixo apenas entre a maioria dos pacientes com dieta líquida, mas também entre aqueles com dieta pastosa e sólida, ressaltando a importância da intervenção nutricional em todo período de tratamento da estenose esofágica.

A maioria dos pacientes apresentava disfagia em variados graus, e os resultados obtidos mostraram a influência da dilatação na remissão da intensidade. Os pacientes com disfagia grave, ou seja, que mantinham dieta de consistência lí- quida, haviam realizado um número menor de sessões de dilatação (mediana=2), e estavam com maior limitação no lúmen esofágico (mediana=7 milímetros) do que os pacientes com disfagia moderada e grave. O limite ideal para a passagem do dilatador em crianças é de 11 milímetros (mm), e de $12,8 \mathrm{~mm}$ para adolescentes ${ }^{27}$, ou seja, a disfagia independe da etiologia da estenose esofágica, mas sua remissão depende do diâmetro do lúmen esofágico após dilatação. Em condições de normalidade, o lúmen esofágico apresenta certa distensibilidade em seu diâmetro, porém, após a instalação da fibrose cicatricial reparadora, há vários graus de diminuição do diâmetro esofágico, sendo necessário o fracionamento da dieta e o aumento da densidade energética, não apenas por o paciente não tolerar grandes volumes, mas pela ocorrência de refluxo gastroesofágico, que nesta condição pode ser intensificado ${ }^{28}$. Mas é relevante considerar o percentual de energia proveniente de lipídeos em relação ao Valor Energético Total (VET) da dieta, não ultrapassando a variação percentual aceitável, pois a dieta hiperlipídica também pode ser um fator determinante no aumento da frequência de sintomas de refluxo gastroesofágico ${ }^{29}$.

Essa acentuada limitação do lúmen esofágico parece ter restringido principalmente a ingestão de porções do grupo de cereais, frutas e 
leguminosas, visto que o consumo foi menor entre os pacientes com dieta líquida. Esse aspecto, uma vez identificado, deve ser reforçado na orientação nutricional, uma vez que é possível ingeri-los na forma líquida e, ainda, acrescentar óleos vegetais à dieta, visando principalmente ao aumento da densidade energética. Porém, os pacientes com dieta líquida consumiram menos de uma porção desse grupo alimentar. No início do tratamento com dilatação endoscópica, o acompanhamento da ingestão alimentar e do estado nutricional deve ser reforçado. Um estudo africano realizado em Serra Leoa ${ }^{14}$, em 2007, constatou que as crianças $(n=24)$ apresentavam disfagia grave e sinais de desnutrição no início do tratamento com dilatação, porém, esta informação foi apenas citada e nenhum método de avaliação ou dado antropométrico foi relatado.

Na dieta líquida houve o predomínio de porções de leite, tanto que não houve diferença estatisticamente significante em relação ao grupo com dieta pastosa e sólida. Contudo, nos três grupos, observou-se uma parcela considerável de pacientes cujo consumo de porções de leite estava abaixo do proposto pela pirâmide alimentar. Talvez a falta de informação dos pais e responsáveis quanto às preparações adequadas, frente à dificuldade em alimentar o paciente devido à presença de disfagia, possa ocasionar uma monotonia alimentar, no qual o leite seja o principal alimento de escolha.

Os resultados mostraram que o consumo de açúcar também deve ser monitorado em dietas de consistência modificada. A média de porções de açúcar consumidas entre os pacientes com dieta líquida não diferiu dos pacientes com dieta pastosa e sólida. No entanto, no grupo com dieta pastosa, embora a maioria de suas características tenha sido semelhante à dieta sólida, observou-se que o consumo de porções do grupo de açúcares foi maior que na dieta sólida, com significância estatística. Um estudo caso-controle realizado na Turquia, que avaliou o desenvolvimento bacteriano e fúngico dentário em crianças com disfagia e estenose esofágica, mostrou que aquelas que consumiam dieta pastosa apresentavam maior risco de desenvolvimento de cárie dentária ${ }^{30}$. Isso sugere que a alta ingestão de açúcar na dieta pastosa é um aspecto que necessita de intervenção nutricional, principalmente no sentido de priorizar o consumo de cereais como fonte de energia, que, ao contrário do açúcar refinado, fornecem outros nutrientes importantes, como aminoácidos, vitaminas e fibras, principalmente quando integrais.

Para avaliar qualitativamente a ingestão alimentar, utilizou-se a pirâmide alimentar, adaptada à população brasileira em 1999 por Philippi et al..$^{25}$ a partir da pirâmide americana, e que pode ser aplicada tanto para adultos quanto para crianças e adolescentes. Porém, especificamente para crianças entre 2 e 3 anos, adotou-se a pirâmide alimentar para esta faixa etária, também desenvolvida por Philippi et al. ${ }^{19} \mathrm{em} 2003$. Isto porque nesta idade as crianças apresentam maior estabilidade no crescimento, o que condiciona diminuição do apetite, e por isso o tamanho recomendado das porções é adaptado às características próprias da idade, às variações do apetite e à limitada capacidade gástrica. Para crianças entre 6 e 24 meses utilizou-se o guia alimentar do Ministério da Saúde e da Organização Pan-Americana da Saúde (OPAS) desenvolvido em conjunto a universidades, que considera o perfil epidemiológico e os hábitos alimentares específicos dessa faixa etária ${ }^{24}$. Assim, as porções de alimentos não são igualmente estabelecidas entre as pirâmides alimentares utilizadas no presente estudo.

Vários métodos são utilizados para avaliar o consumo alimentar a fim de se obterem dados válidos, reprodutíveis e comparáveis. A seleção do método depende dos objetivos do estudo, da população-alvo, bem como dos recursos disponíveis e exequíveis. Nesse contexto, o recordatório de 24 horas foi o instrumento de escolha neste estudo, pois além de refletir a ingestão atual, tem a vantagem de ser rápido e seguro, pois é baseado na memória recente dos indivíduos, porém, como desvantagem, exclui as variações diárias da inges- 
tão alimentar22,23,31. Erros podem ocorrer em estudos que avaliam a ingestão alimentar de apenas um dia, pois os indivíduos não consomem os mesmos alimentos diariamente. Por este motivo, é recomendado o emprego do recordatório de 24 horas por mais dias para avaliar o total da ingestão de alimentos e nutrientes ${ }^{22,32-34}$. Porém, como a estenose esofágica cursa com limitada ingestão alimentar, a população-alvo recebeu orientação nutricional já na primeira consulta, e assim, considerou-se inapropriado reaplicar o recordatório de 24 horas, ou ainda solicitar registro alimentar, pois neste caso, a orientação nutricional realizada na primeira consulta constituiria um viés de erro na análise da ingestão alimentar, visto que este é o objetivo principal do estudo.

Crianças e adolescentes com estenose esofágica devem receber acompanhamento nutricional, ressaltando que, mesmo com inúmeras recorrências de disfagia durante o tratamento da estenose esofágica, é possível que o paciente tenha uma adequada ingestão alimentar, balanceada, que atenda às suas necessidades energéticas e de nutrientes, permitindo a manutenção do estado nutricional, crescimento e desenvolvimento, e menor morbidade inerente ao processo estenótico e ao respectivo tratamento. O acompanhamento do estado nutricional e da ingestão alimentar é de extrema importância no tratamento de pacientes com estenose esofágica, principalmente daqueles com disfagia grave, que estão no início do tratamento com dilatação, cuja dieta deve ser adaptada à consistência líquida, devido ao risco nutricional que se atribui à limitada ingestão principalmente de cereais, leguminosas, e óleos e gorduras, que pode refletir em baixa densidade energética da dieta e inúmeras deficiências nutricionais.

São necessários estudos longitudinais na faixa etária pediátrica devido à escassez de estudos na literatura científica a respeito da melhor abordagem nutricional de um quadro patológico que culmina diretamente na limitação da ingestão alimentar.

\section{COLABORADORES}

Todos os autores participaram de todas as fases do artigo.

\section{REFERÊ NCIAS}

1. Pereira-Lima JC, Marroni C, Cheinquer N, Marques DL, Hornos AP, Galant L, et al. Dilatação endoscópica de esôfago sem o auxílio de fluoroscopia: experiência em 1358 sessões. GED. 2002; 21(5): 201-6.

2. Michaud L, Guimber D, Sfeir R, Rakza T, Bajja H, Bonnevalle $M$, et al. Sténose anastomotique après traitement chirurgical de l'atrésie de l'aesophage: fréquence, facteurs de risque et efficacité dês dilatations aesophagiennes. Arch Pédiatr. 2001; 8(3):268-74.

3. Chittmittrapap S, Spitz L, Kliely ME, Brereton RJ. Anastomotic stricture following repair of esophageal atresia. J Pediatr Surg. 1990; 25(5): 508-11.

4. Van der Zee VD, Bax KN. Thoracoscopic treatment of esophageal atresia with distal fistula and of tracheomalacia. Semin Pediatr Surg. 2007; 16(4): 224-30

5. O’Neill JA, Betts J, Ziegler MM, Schnaufer L, Bishop $\mathrm{HC}$, Templeton J. Surgical management of reflux strictures of the esophagus in childhood. Ann Surg. 1982; 196(4):453-9.

6. Rode H, Millar AJ, Brown RA, Cywes S. Reflux strictures of the esophagus in children. J Pediatr Surg. 1992; 27(4):462-5.

7. Numanoglu A, Millar AJ, Brown RA, Rode H. Gastroesophageal reflux strictures in children, management and outcome. Pediatr Surg Int. 2005; 21(8):631-4.

8. Poley JW, Steyerberg EW, Kuipers EJ, Dees J, Hartmans R, Tilanus HW, et al. Ingestion of acid and alkaline agents: outcome and prognostic value of early upper endoscopy. Gastrointest Endosc. 2004; 60(3):372-77.

9. Doワan Y, Erkan T, Çokugras FÇ, Kutlu T. Caustic gastroesophageal lesion in childhood: analysis of 473 cases. Clin Pediatr. 2006; 45(5):435-8.

10. Casasnovas $A B$, Martinez $E E$, Cives RV, Jeremias AV, Sierra RT, Cadranel S. A retrospective analysis of ingestion of caustic substances by children: tenyear statistics in Galicia. Eur J Pediatr. 1997; 156(5): 410-4.

11. Mamede RCM, Filho FVM. Ingestion of caustic substances and its complications. São Paulo Med J. $2001 ; 119(1): 10-5$. 
12. Lamireau T, Llanas B, Deprez C, Hammar FE, Vergnes $P$, Demarquez $J$, et al. Gravité des ingestions de produits caustiques chez l'enfant. Arch Pediatr. 1997; 4(6):529-34.

13. Hodge GE, Scharfe EE. Strictures of the esophagus. Can Med Assoc J. 1937; 37(6):541-7.

14. Contini S, Tesfaye M, Picone P, Pacchione D, Kuppers B, Zambianchi C, et al. Corrosive esophageal injures in children: a shortlived experience in Sierra Leone. Int J Pediatr Otorhinolaryngol. 2007; 71(10):1597-604.

15. Ko HK, Shin JH, Song HY, Kim YJ, Ko GY, Yoon HK. Balloon dilation of anastomotic strictures secondary to surgical repair of esophageal atresia in a pediatric population: long-term results. J Vasc Interv Radiol. 2006; 17(8):1327-33.

16. Güitrón A, Adalid R, Nares J, Mena G, Gutiérrez JÁ, Olivares C. Estenosis esofágica benigna en pacientes lactantes y preescolares. Resultados de dilatación endoscópica. Rev Gastroenterol Mex. 1999; 64(1):12-5.

17. Hiley SA, Attwood SEA. Guidelines on the use of oesophageal dilatation in clinical practice. Gut. 2004; 53(1):11-6.

18. Kaiser LL, Melgar-Quiñonez HR, Lamp CL, Johns MC, Sutherlin JM, Harwood JO. Food security and nutritional outcomes of preschool-age Mexican-American children. J Am Diet Assoc. 2002; 102(7): 924-9.

19. Philippi ST, Cruz ANT, Colucci AMA. Pirâmide alimentar para crianças de 2 a 3 anos. Rev Nutr. 2003; 16(1):5-19. doi: 10.1590/S1415-5273200300100 002.

20. Barbosa RMS, Croccia C, Carvalho CGN, Franco VC, Salles-Costa R, Soares EA. Consumo alimentar de crianças com base na pirâmide alimentar brasileira infantil. Rev Nutr. 2005; 18(5):633-41. doi: 10.1590/S1514-52732005000500006.

21. Fulgoni V, Nicholls J, Reed A, Buckley R, Kafer K, Huth P. Dairy consumption and related nutrient intake in African-American adults and children in the United States: continuing survey of food intakes by individuals 1994-1996, 1998, and the National Health And Nutrition Examination Survey 1999-2000. J Am Diet Assoc. 2007; 107(2):256-64.

22. Freudenheim JL. A review of study designs and methods of dietary assessment in nutritional epidemiology of chronic disease. J Nutr. 1993; 123(2):401-5.

23. Thompson FE, Byers T. Dietary assessment resource manual. J Nutr. 1994; 124(11):2245-317.
24. Monte CMG, Giugliani ERJ, Carvalho MFCC, Philippi ST, Albuquerque ZPA. Guia alimentar para crianças menores de dois anos Brasília: Ministério da Saúde; 2002. p.87-97. Série A. Normas e Manuais Técnicos, 107.

25. Philippi ST, Latterza AR, Cruz ATR, Ribeiro LC. Pirâmide alimentar adaptada: guia para escolha dos alimentos. Rev Nutr. 1999; 12(1):65-80. doi: 10.15 90/S1514-52731999000100006.

26. Quinton A. Jandel SigmaStat: Scientific statistical software, version 2.0. San Rafael (California); 1995.

27. Ferguson DD. Evaluation and management of benign esophageal strictures. Dis Esophagus. 2005; 18(6):359-64.

28. Pehl C, Pfeiffer A, Waizenhoefer A, Wendl B, Schepp W. Effect of caloric density of a meal on lower oesophageal sphincter motility and gastrooesophageal reflux in healthy subjects. Aliment Pharmacol Ther. 2001; 15(2):233-9.

29. Fox M, Barr C, Nolan S, Lomer M, Anggiansah A, Wong T. The effects of dietary fat and calorie density on esophageal acid exposure and reflux symptoms. Clin Gastroenterol Hepatol. 2007; 5(4):439-44.

30. Oncag O, Alpoz AR, Eronat C. Salivary Streptococcus mutans, Lactobacilli levels and buffer capacity in children with esophageal burns. J Clin Pediatr Dent. 2000; 24(2):147-51.

31. Gersovitz M, Madden JP, Smiciklas-Wright $H$. Validity of the 24-hr dietary recall and seven-day record for group comparisons. J Am Dietetic Assoc. 1978; 73(1):48-55.

32. Sempos CT, Johnson NE, Smith EL Gilligan C. Effects of intraindividual and interindividual variation in repeated dietary records. Am J Epidemiol. 1985; 121(1):20-30

33. Palaniappan U, Cue RI, Payette H, Gray-Donald K. Implications of day-to-day variability on measurements of usual food and nutrient intakes. J Nutr. 2003; 133(1):232-5.

34. Cavalcante AAM, Priore SE, Franceschini SCC. Estudos de consumo alimentar: aspectos metodológicos gerais e o seu emprego na avaliação de crianças e adolescentes. Rev Bras Saúde Matern Infant. 2004; 4(3):229-40.

Recebido em: 23/10/2008

Versão final reapresentada em: 29/6/2010 Aprovado em: 16/12/2010 
